\title{
Vanishing Density of States in Weakly Disordered Weyl Semimetals
}

\author{
Michael Buchhold, ${ }^{1}$ Sebastian Diehl, ${ }^{2}$ and Alexander Altland ${ }^{2}$ \\ ${ }^{1}$ Department of Physics and Institute for Quantum Information and Matter, \\ California Institute of Technology, Pasadena, California 91125, USA \\ ${ }^{2}$ Institute for Theoretical Physics, Universität zu Köln, D-509237 Köln, Germany
}

(Received 6 May 2018; published 19 November 2018)

\begin{abstract}
The Brillouin zone of the clean Weyl semimetal contains points at which the density of states (DOS) vanishes. Previous work suggested that below a certain critical concentration of impurities this feature is preserved including in the presence of disorder. This result got criticized for its neglect of rare disorder fluctuations which might bind quantum states and hence generate a finite DOS. We here show that in spite of their existence these states are so fragile that their contribution effectively vanishes when averaged over continuous disorder distributions. This means that the integrity of the nodal points remains protected for weak disorder.
\end{abstract}

DOI: 10.1103/PhysRevLett.121.215301

Introduction.-The three dimensional Weyl semimetal is a paradigm of gapless topological quantum matter. Its defining feature is the presence of an even number of topologically protected band touching points in the Brillouin zone. The linearly dispersive behavior of these Weyl points is attracting a lot of attention and has put the system at the center of experimental [1-6] and theoretical [7-12] studies of relativistic Fermi matter in solid state physics contexts.

While individual Weyl nodes enjoy topological protection-they can be moved in the Brillouin zone but not individually destroyed - the presence of singular band touching points makes the system highly susceptible to perturbations away from the clean, noninteracting limit. Specifically, the role played by static disorder has been the subject of a partly controversial debate: renormalized diagrammatic perturbation theory in $d=2+\epsilon$ dimensions [13-20] evaluated at $\epsilon=1[21,22]$, and the mean field analysis of a nonlinear sigma model approach [13,14,23,24] predict the existence of a critical disorder strength $K_{c}$, below which disorder is irrelevant and the system behaves effectively clean at large length scales. However, this finding is at variance with a complementary approach [25] arguing that rare disorder configurations are capable of generating zero energy states, leading to a finite density of states at the Dirac point. No matter how small, this would rule out the density of states (DOS) as an order parameter and compromise the existence of a phase transition driven by disorder strength. Finally, the numerical analysis of the problem is met with various challenges. For example, lattice implementations categorically model even numbers of Weyl nodes, which in the presence of finite range correlated disorder are coupled. This makes it difficult to resolve the spectrum of individual nodes down to the lowest energies, and the current status [26-36] does not appear to be fully conclusive.
In this Letter we analytically demonstrate that the nodal DOS in the weakly disordered Weyl semimetal remains vanishing, including if rare fluctuations are taken into account. At first sight this may sound counterintuitive. Rare fluctuations include configurations in which the chemical potential of the system is effectively lowered (or raised) over sizable regions in space. One might expect this to shift the Weyl cone away from the stateless nodal point with vanishing DOS and effectively accumulate finite spectral weight at zero energy. In the following, we will analyze three different model setups to demonstrate that this is not what is happening. Specifically, we will (a) analytically compute the DOS of a box potential mimicking rare event fluctuations, (b) apply the large fluctuation stationary phase methods to compute the DOS of rare Gaussian fluctuations, and (c) consider a $T$-matrix approach to the DOS of multiple pointlike impurities. While these setups lead to distinct spectral density profiles and call for different computational schemes they all have in common that the spectral density at the nodal points remains vanishing.

Box potential.- To start with consider a spherical potential of radius $b$ and uniform depth $\lambda$ as a cartoon of a rare configuration. Unlike in a Schrödinger problem, such wells bind states only at zero energy and only for depths, $\lambda_{c} \equiv m \pi / b$, where $m$ is an integer $[37,38]$. Away from these singular configurations, the fragile bound states turn into scattering states whose interplay with the continuum of extended states is key to the understanding of the DOS: according to the Levinson theorem (a close cousin of the Friedel sum rule) $[39,40]$, the number of bound states in a scattering setup equals the phase shift difference, $(2 / \pi) \int d \omega \partial_{\omega} \delta(\omega)$ accumulated by all scattering states. (In the Weyl problem, the integral extends over positive and negative energies, up to a cutoff beyond which the potential 
is no longer effectively seen.) This implies that no additional DOS is generated by a potential and that accumulations of density of states in one energy region, i.e., by a bound state or resonance, are screened by a diminished scattering state $\delta \nu(\omega)=(2 / \pi) \partial_{\omega} \delta(\omega)$ elsewhere.

The explicit diagonalization of the potential well Hamiltonian [37,39] shows that for $\lambda=\lambda_{c}$ the phase drop $\pi \rightarrow 0$ compensating for the zero energy bound state is concentrated in the infinitesimal neighborhood of zero energy. In effect, this means that no spectral weight accumulates there. Tuning away from the singular value, $\lambda_{c}$, the bound state becomes a finite energy scattering state. However, the ensuing narrow peak $\delta \nu(\omega)$ is screened by a negative background (see Fig. 1), in quantitative agreement with the sum rule. The explicit calculation [40] shows that the balance is such that $\nu(0)=0$ for all parameter values: a potential well does generate spectral density, but never at zero energy. This is the reason for the absence of a rare event contribution to the DOS in the Weyl problem. One might object that the argument makes reference to a potential well of specific shape (a box) and does not account for potential side effects due to correlations between neighboring potential inhomogeneities. In the following, we will show that the vanishing of the DOS is robust and survives these generalizations.
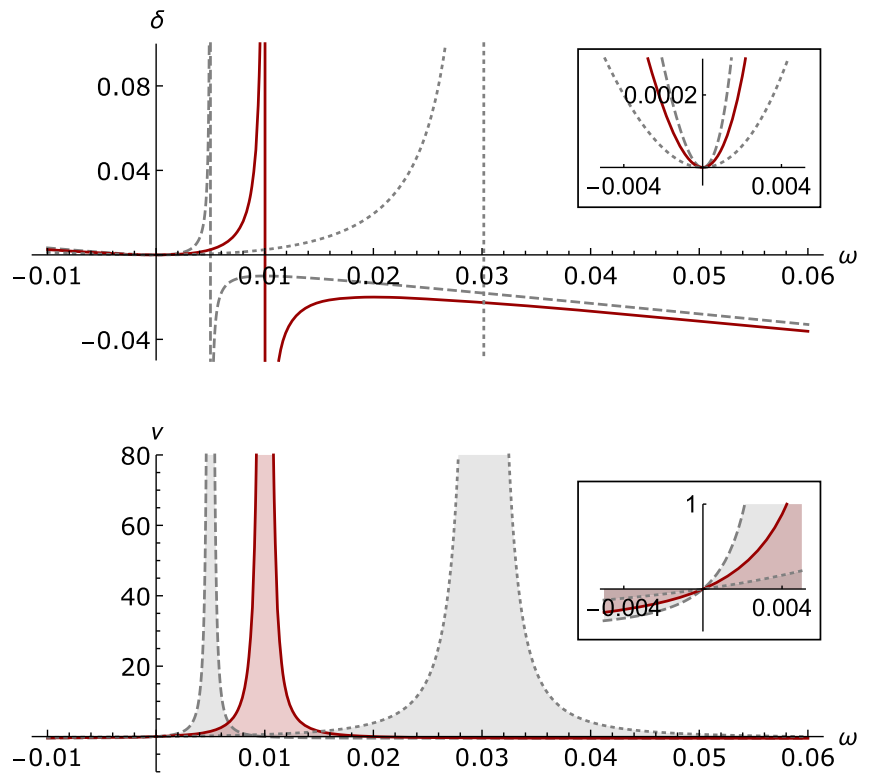

FIG. 1. Top: phase shift $\delta(\omega)$ for three configurations, $\lambda=\pi / b+\Delta, \Delta=0.01$ (dashed curve), 0.02 (solid curve), 0.06 (dotted curve). With decreasing $\Delta$, a resonance of width $\sim \Delta$ moves closer to the origin. At the origin, $\delta(0)=\delta^{\prime}(0)=0$ touches zero (inset). Bottom: the corresponding shift in the DOS $\delta \nu \equiv \nu_{\lambda}-\nu_{0}$ compared to the clean Weyl problem assumes the form of narrow peaks. Their positive spectral weight is screened by a negative background in such a way that $\nu(0)=0$, always (inset). This includes the limit, $\Delta \rightarrow 0$, in which the singular derivative of the phase shift reflects the presence of a bound state.
Random potential wells.-We follow Ref. [25], and analyze the rare event DOS in a finite range Gaussian correlated potential via a standard [41-46] large deviation analysis. Referring to Ref. [40] for details, we limit ourselves to a sketch of the construction and explain where we deviate from Ref. [25]. The starting point is a representation of the DOS as a Gaussian functional integral

$\nu(\omega)=-\frac{1}{2 \pi L^{3}} \operatorname{Im} \int \mathcal{D}[\bar{\psi}, \psi] \int(\bar{\phi} \phi-\bar{\chi} \chi)\left\langle e^{i S[\psi]}\right\rangle$,

where $S[\psi] \equiv \int \bar{\psi}\left(\omega^{+}-\hat{H}\right) \psi, \psi=(\phi, \chi)^{T}$ is a field comprising commuting and anticommuting components, $\phi$ and $\chi$, respectively, and $\hat{H}=-i v_{0} \sigma_{i} \partial_{i}+V_{x}$ contains the WeylHamiltonian with velocity $v_{0}$ and a Gaussian potential, $\left\langle V_{x} V_{x^{\prime}}\right\rangle=W^{2} \exp \left(-\left|x-x^{\prime}\right| / \xi\right)$ of strength $W$ and correlation length, $\xi$, mimicking the formation of finite range potential wells.

The auxiliary integration over the anticommuting $\chi$ is required to avoid the unwanted appearance of determinants $\operatorname{det}\left(\omega^{+}-\hat{H}\right)$ after the integration [47]. In this case, the average over disorder simply generates an effective action

$S_{\mathrm{eff}}[\psi]=\int_{x} \bar{\psi}_{x}\left(\omega^{+}+i v_{0} \sigma_{i} \partial_{i}+i W^{2} \int_{y} e^{-\xi^{-1}|x-y|} \bar{\psi}_{y} \psi_{y}\right) \psi_{x}$,

quartic in integration variables. We are now at the crossroad where the two principal approaches to computing the DOS part: where Refs. $[14,16]$ apply renormalized perturbation theory to the analysis of the quartic nonlinearity, the starting point of Ref. [25] is the observation [43-45] that the physics of rare events is contained in inhomogeneous "instanton" solutions to the variational equations of the action (2).

Following the second approach, we consider nontrivial solutions $\psi_{I}$, of the variational equation $\delta_{\psi} S=0$ identified in Ref. [25]. Referring to the original reference and Ref. [40] for details we note that a class of instanton solutions with regular behavior at the origin and power law decay at large scales, $r>\xi$ can be identified. Reference [25] reasoned that, to exponential accuracy, the value of the DOS close at zero energy should be determined by the instanton action, $\nu \sim \exp \left(-S\left[\psi_{I}\right]\right) \sim \exp \left[-v_{0}^{2} /(W \xi)^{2}\right]$. We here take the additional step to include quadratic fluctuations around the instanton saddle point. That fluctuations may be less innocent than in conventional large deviation phenomena is indicated by the fragility of the bound states discussed above: one may suspect that in spite of the finite probability to find bound states, their singular sensitivity to parameter variations leads to a vanishing measure. Within an integral approach, this would show in a vanishing fluctuation contribution around extremal configurations.

The extremal solutions $\psi_{I}$ break seven continuous symmetries: translational invariance in three directions, 
three independent rotations in the complex two-component Weyl space, and one supersymmetry. While the first six are harmless and can be treated according to standard procedures in instanton calculus [48], the supersymmetry breaking deserves more attention: consider a commuting solution of the stationary equations, $\psi_{I}=\left(\phi_{I}, 0\right)$, where $\phi_{I}$ is a twocomponent complex Weyl spinor. If we act on this configuration with a rotation in super space, $\psi_{I} \rightarrow W \psi_{I}$, where $W=\exp \left(\begin{array}{cc}0 & \eta \rrbracket \\ \bar{\eta} & 0\end{array}\right), \mathbb{1}$ is the unit-matrix in Weyl space, and $\eta, \bar{\eta}$ are Grassmann variables, a new solution is generated. (The matrix $W$ commutes with the Hamiltonian, which is the supersymmetry.) This means that $\eta, \bar{\eta}$ are Grassmann-zero modes, and that the generalization $\eta \rightarrow \eta(x)$ to fields with slow coordinate dependence generates soft fluctuation modes. The next step in the analysis is to expand the action around $\psi_{I}$ to at least quadratic order in fluctuation modes. Expanding a general Grassmann fluctuation as $\eta(x)=$ $\sum_{a} F_{a}(x) \eta_{a}$ in a set of suitably defined functions, $F_{a}$, this leads to an expression of the symbolic structure $S[\eta]=$ $\sum_{a} \bar{\eta}_{a} X_{a b} \eta_{b}$ governed by an effective fluctuations operator. After integration over $\eta_{a}$ the integral picks up a factor $\operatorname{det}(X)$ which, unlike with commuting fluctuation variables, appears in the numerator of the fluctuation prefactor. The operator $X$ contains at least one zero eigenvalue, whose eigenmode is the constant fluctuations $\eta_{0}=$ const. In the computation of the density of states this factor is canceled after the expansion of the preexponential term $\bar{\psi} \psi$ in the Grassmann variables $\left\{\eta_{a}\right\}$ and integration. This $0 / 0$ cancellation is of a general nature and safeguards the correct normalization of observables [47]. However, should a fluctuation operators contain $n>1$ one zero eigenvalues, the integration leads to a factor $0^{n} / 0$ and a vanishing result.

The setting as described so far is of general nature and in the same way applies to, e.g., a disordered Schrödinger operator. What makes the Weyl problem special is that at $\omega=0$ it indeed possesses an extensive (diverging in the limit of infinite volume) number of fluctuation modes $\eta_{a}$ of vanishing action, and the above structure implies a vanishing of the rare event contribution to the DOS. The existence of these modes simply follows from the fact that the Weyl operator is of first order in derivatives. The condition of vanishing fluctuation action, thus assumes the form $\hat{O} \eta=0$, where $\hat{O}$ is a first order partial differential operator. Unlike with equations governed by second order elliptic operators (as in a Schrödinger problem) these first order differential equations possess an extensive number of solutions which can be found, e.g., via the method of characteristics [40]. In the asymptotic limit of a perfectly linear Weyl operator and a single instanton defined in a box of unbounded extension, $L \rightarrow \infty$ we indeed obtain a diverging number of zero fluctuation zero modes, and hence a vanishing DOS. The large spatial support of these fluctuation modes reflects the absence of compact exponentially bound eigenstates, as exemplified above for the box potential.
A realistic Weyl operator is not perfectly linear but contains higher order derivatives due to, e.g., an underlying lattice dispersion. (Higher order derivatives in the fluctuation action are also induced via the coupling between the zero modes $\eta$ to other fluctuation modes.) We also have to account for multi-instanton solutions containing the superposition of inhomogeneities $\psi_{I}$ centered around different coordinates. These generalizations affect the above analysis via the appearance of an effective length scale, $L \sim \min \left(L_{I}, \Gamma^{-1}\right)$, loosely to be identified with the instanton separation, $L_{I}$ or the inverse of momentum scale, $\Gamma$, above which the spectrum ceases to be linear. The introduction of this scale lifts the zero degeneracy of the fluctuation modes to a spectrum $\epsilon_{n} \sim L^{-1} F(n)$, where $F(n)$ is a polynomial factor containing some 'quantum numbers' $\left\{n_{i}\right\}$ describing the angular momentum and radial quantization of the fluctuation modes. The fluctuation determinant contains the product $\prod_{n} \epsilon_{n}$, and a quick estimate shows that the product up to values $\epsilon_{n} \sim 1$ leads to a factor $\sim \exp (-c L)$, where $c=\mathcal{O}(1)$. This factor sets an upper bound for the DOS, as obtained by the present approach. For example, if $L \sim \exp \left(S_{I}\right)$ is identified with the expected separation between instanton configurations, we obtain a bound $\sim \exp \left[-c \exp \left(v_{0}^{2} / W^{2} \xi^{2}\right)\right]$ double exponential in the disorder concentration. To summarize, the inclusion of fluctuations leads to a drastic suppression of the DOS compared to the fluctuationless stationary phase analysis. However, in view of approximative assumptions-linearity of the spectrum, neglect of correlations-required for the computation of fluctuation determinants, the DOS cannot be determined with ultimate precision.

Multiple impurities.-We now discuss a model that does account for potential correlations and does tolerate (even require) nonlinear dispersion. The price to be payed for the enhanced degree of generality is that the potential landscape is modeled in a simplistic fashion: following Ref. [49], we consider a system of $N$ pointlike impurities, $V(\mathbf{r})=\sum_{i} U_{i} \delta\left(\mathbf{r}-\mathbf{r}_{i}\right)$ at positions $\mathbf{r}_{i}$ and strength $U_{i}$. Here, the $\delta$ potentials are a cartoon of fluctuations whose range $\xi>b^{-1}$ is larger than the momentum separation between different Weyl nodes so that transnodal scattering is excluded, and at the same time $\xi<M^{-1}$ smaller than an effective large momentum cutoff of individual nodes. Within this parametric window, the spatial profile of individual minima is not resolved and a pointlike model justified. At the same time, the model is simple enough to be exactly solvable by $T$-matrix techniques.

An expansion of the Green function $\hat{G}=\left(\omega^{+}-\hat{H}_{0}-\right.$ $\hat{V})^{-1}$ shows that the impurity contribution to the DOS is given by $\delta \nu(\omega)=-(1 / \pi) \operatorname{Imtr}\left[\hat{G}_{0}\left(1-\hat{V} \hat{G}_{0}\right)^{-1} \hat{V} \hat{G}_{0}\right]$, where $\hat{G}_{0}$ is the clean Green function. Introducing a diagonal matrix $U=\operatorname{diag}\left(U_{1}, \ldots, U_{N}\right)$ and the projection operator $\hat{P}=\sum_{i}\left|\mathbf{r}_{i}\right\rangle\left\langle\mathbf{r}_{i}\right|$ onto impurity coordinates, this can be represented as $\delta \nu(\omega)=-(1 / \pi) \partial_{\omega} \operatorname{Im} F(\omega)$, where the 
"free energy" $F(\omega)=\ln \operatorname{det}\left(\hat{U}^{-1}-\hat{G}_{0}\right)$ contains the projected Green function $\hat{G}_{0}=\hat{P} G_{0} \hat{P}$. In this way, the computation of the DOS is reduced to that of the determinant of an $N \times N$ matrix. The projected Green function matrix elements, $G\left(\mathbf{r}_{i}-\mathbf{r}_{j}\right)$ featuring in this matrix contain the singular diagonals, $G(0)=\int\left(d^{3} k /(2 \pi)^{3}\right)\left[\left(\omega^{+}-\right.\right.$ $\left.\left.k_{i} \sigma_{i}\right) /\left(\omega^{+2}-k^{2}\right)\right]$ (we set $\nu_{0} \equiv 1$ here). While this expression can be regularized in different ways, the introduction of nonlinearity in the band dispersion, $\epsilon(k)=v|k|+$ $\mathcal{O}\left(k^{2} / M^{2}\right)$, where $M$ is an effective large momentum cutoff, may be the most natural for a system defined on a lattice. Either way, one obtains $G(0)=-\left[\left(\omega^{+}(M+\right.\right.$ $i \omega)) / 4 \pi]+\mathcal{O}\left(\omega^{2}\right)$. The off-diagonal elements are nonsingular, and for small $\omega$ assume the form $G(\mathbf{r})=$ $-\left[\left(i r_{i} \sigma_{i}+r^{2} \omega\right) /\left(4 \pi r^{3}\right)\right]+\mathcal{O}\left(\omega^{2}\right)$.

It is instructive to explore the ensuing DOS profile for a system of just two impurities at distance $r$. In this case, $F(\omega)=-2 \ln \left[\left(U_{1}^{-1}+M \omega^{+}\right)\left(U_{2}^{-1}+M \omega^{+}\right)-r^{-4}-r^{-2} \omega^{+2}\right]$ and the DOS near zero is obtained as the asymptotically linear function $\delta \nu(\omega)=\left[\left(r^{4}\left(U_{1}+U_{2}\right) \omega\right) /\left(\pi^{2}\left(U_{1} U_{2}-\right.\right.\right.$ $\left.\left.\left.r^{4}\right)\right)\right]+\mathcal{O}(\omega)^{2}$. Away from zero energy the DOS shows resonances at energies $\omega_{0}=-\left(1 / 2 M U_{1} U_{2}\right)\left\{U_{1}+U_{2} \pm\right.$ $\left.\left[\left(U_{1}-U_{2}\right)^{2}+4 r^{-4} U_{1}^{2} U_{2}^{2}\right]^{1 / 2}\right\}$, which are the resonant energies $1 / M U_{i}$ of the isolated impurities, shifted by an $r$-dependent hybridization energy. The peak values at resonance diverge in $M$ and the width shrinks in the inverse of the same parameter. Each resonance carries unit spectral weight, as indicated in the right inset of Fig. 2, where the integrated spectral density $N_{\epsilon} \equiv$ $\int_{\omega_{0}-\epsilon}^{\omega_{0}+\epsilon} d \omega \delta \nu(\omega)=-\left.(1 / \pi) \operatorname{Im} F(\omega)\right|_{\omega_{0}-\epsilon} ^{\omega_{0}+\epsilon}$ is shown as a dashed curve.

Setting $\omega_{0} \rightarrow 0$, we identify the configurations $U_{1} U_{2}=$ $r^{4}$ for which the impurity hybridization pushes the resonance centers to zero. In this limit, the slope of the DOS $\partial_{\omega} \delta \nu(\omega)$ diverges and $\lim _{\omega \rightarrow 0} \delta \nu(\omega)$ is no longer defined. However, the integrated spectral weight $N_{\epsilon}$ is still well defined and inspection of the logarithm shows that $N_{\epsilon} \rightarrow 0$

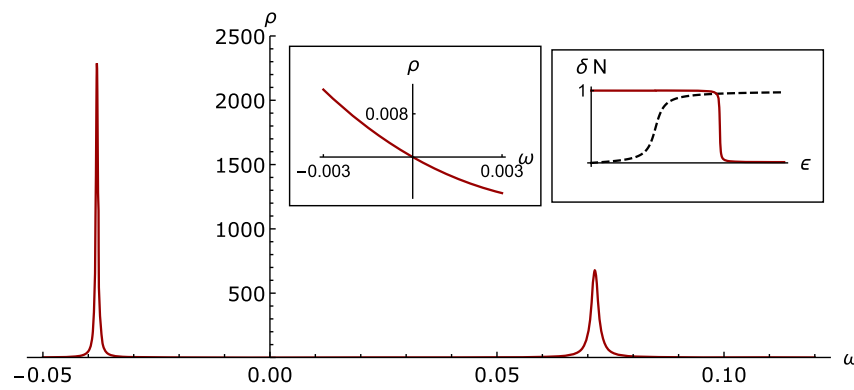

FIG. 2. Exemplary DOS of a system of two impurities $\left(r=15 / M, U_{1}=-75 / M^{2}, U_{2}=150 / M^{2}\right.$ in the units discussed in the text). Left inset: enlargement of the DOS near zero energy. Right inset: dashed curve, integrated spectral weight around the resonance centers; solid curve, the same for a resonance at zero energy. for energies $\epsilon \gtrsim \epsilon_{0} \equiv\left(U_{1}^{-1}+U_{2}^{-1}\right) / M$. This is shown in the right inset Fig. 2, where the position of the final kink is set by $\epsilon_{0}$. The structure indicates that the DOS carried by the zero energy peak is "screened" by an equally strong counterweight in its immediate vicinity, $\epsilon_{0} \sim M^{-1}$. In the asymptotic limit of a fully linear spectrum, $M \rightarrow \infty$, the zero energy resonance does not carry spectral weight at all, $\delta N_{\epsilon}=0$, and for finite $M, \delta N_{\epsilon}$ shows sign-fluctuating singular behavior at energies $\omega \lesssim \epsilon_{0}(M)$. However, regardless of the value of $M$, the above singular profile will never be realized in any specific sample of impurity potentials. The reason is that upon approaching zero energy the resonant peaks not only become larger they also become narrower. A careful statistical analysis of the problem [40] shows that the limit of a singular zero energy resonance is an event of measure zero in the sense of probability theory, and is strictly nonobservable.

Summary and outlook.-Summarizing, we have analyzed three different models of disorder in Weyl semimetals, all preserving the integrity of the nodal point. Individual of these models emphasized different facets of the problem. Specifically, the box potential model was simple enough to be amenable to the exact analytic solution by scattering methods. The model of Gaussian distributed disorder could no longer be solved rigorously, in exchange for a more realistic modeling of a smooth disorder landscape. Finally, the multi-impurity model described individual impurities in simplistic ways, but added the effects of impurity correlations and spectral curvature to the analysis. The fact that three different models and different analytic approaches lead to identical conclusions indicates that the protection of the nodal structure is a general result.

We have seen that in all three models the DOS away from zero energy is carried by a peculiar set of resonances [50]. While nothing prevents these resonances from approaching zero, they become narrower (and hence more difficult to observe) in the process. For any finite separation from zero, the nodal DOS remains continuous and vanishing. Only in the limit, the competition of diverging resonance height and vanishing width leads to a singularity. However, this limit has zero statistical measure and is not realized in any specific sample (much like a mathematical zero will not be drawn in any random sampling of real numbers.) This is how the seeming contradiction between zero nodal DOS and resonant DOS elsewhere gets resolved. The detailed analysis of the ensuing statistical DOS distribution is a subject of Ref. [40].

Finally, the principal result of preserved nodal points is a result of conceptual significance. It suggests that the mean field result of a threshold concentration separating a weak and a strong disorder phase survives the presence of rare events, and that there is a genuine phase transition with $\nu(0)$ as its order parameter.

We thank P. W. Brouwer, V. Gurarie, R. Nandkishore, L. Radzihovsky, G. Refael, B. Sbierski, J. H. Wilson, 
K. Ziegler, and M. Zirnbauer for discussions. Work supported by the German Research Foundation (DFG) through the Institutional Strategy of the University of Cologne within the German Excellence Initiative (ZUK 81) and CRC/TR 183-Entangled states of matter (project A02). M. B. acknowledges support from the Alexander von Humboldt foundation.

[1] S.-Y. Xu et al., Science 349, 613 (2015).

[2] B. Q. Lv, H. M. Weng, B. B. Fu, X. P. Wang, H. Miao, J. Ma, P. Richard, X. C. Huang, L. X. Zhao, G. F. Chen, Z. Fang, X. Dai, T. Qian, and H. Ding, Phys. Rev. X 5, 031013 (2015).

[3] H. Inoue, A. Gyenis, Z. Wang, J. Li, S. W. Oh, S. Jiang, N. Ni, B. A. Bernevig, and A. Yazdani, Science 351, 1184 (2016).

[4] S.-Y. Xu et al., Nat. Commun. 6, 7373 (2015).

[5] I. Belopolski et al., Nat. Commun. 7, 13643 (2016).

[6] N. Xu, H. M. Weng, B. Q. Lv, C. E. Matt, J. Park, F. Bisti, V. N. Strocov, D. Gawryluk, E. Pomjakushina, K. Conder, N. C. Plumb, M. Radovic, G. Autès, O. V. Yazyev, Z. Fang, X. Dai, T. Qian, J. Mesot, H. Ding, and M. Shi, Nat. Commun. 7, 11006 (2016).

[7] A. C. Potter, I. Kimchi, and A. Vishwanath, Nat. Commun. 5, 5161 (2014).

[8] P. J. W. Moll, N. L. Nair, T. Helm, A. C. Potter, I. Kimchi, A. Vishwanath, and J. G. Analytis, Nature (London) 535, 266 (2016).

[9] S. A. Parameswaran, T. Grover, D. A. Abanin, D. A. Pesin, and A. Vishwanath, Phys. Rev. X 4, 031035 (2014).

[10] X. Wan, A. M. Turner, A. Vishwanath, and S. Y. Savrasov, Phys. Rev. B 83, 205101 (2011).

[11] F. D. M. Haldane, arXiv:1401.0529.

[12] N. P. Armitage, E. J. Mele, and A. Vishwanath, Rev. Mod. Phys. 90, 015001 (2018).

[13] E. Fradkin, Phys. Rev. B 33, 3263 (1986).

[14] E. Fradkin, Phys. Rev. B 33, 3257 (1986).

[15] P. Goswami and S. Chakravarty, Phys. Rev. Lett. 107, 196803 (2011).

[16] S. V. Syzranov, L. Radzihovsky, and V. Gurarie, Phys. Rev. Lett. 114, 166601 (2015).

[17] S. Syzranov, V. Gurarie, and L. Radzihovsky, Ann. Phys. (Amsterdam) 373, 694 (2016).

[18] B. Sbierski, K. A. Madsen, P. W. Brouwer, and C. Karrasch, Phys. Rev. B 96, 064203 (2017).

[19] B. Roy and S. Das Sarma, Phys. Rev. B 90, 241112 (2014).

[20] B. Roy and S. Das Sarma, Phys. Rev. B 93, 119911(E) (2016).

[21] S. V. Syzranov, V. Gurarie, and L. Radzihovsky, Phys. Rev. B 91, 035133 (2015).
[22] S. V. Syzranov, P. M. Ostrovsky, V. Gurarie, and L. Radzihovsky, Phys. Rev. B 93, 155113 (2016).

[23] A. Altland and D. Bagrets, Phys. Rev. Lett. 114, 257201 (2015).

[24] A. Altland and D. Bagrets, Phys. Rev. B 93, 075113 (2016).

[25] R. Nandkishore, D. A. Huse, and S. L. Sondhi, Phys. Rev. B 89, 245110 (2014).

[26] B. Sbierski, E. J. Bergholtz, and P. W. Brouwer, Phys. Rev. B 92, 115145 (2015).

[27] B. Sbierski, G. Pohl, E. J. Bergholtz, and P. W. Brouwer, Phys. Rev. Lett. 113, 026602 (2014).

[28] J. H. Pixley, D. A. Huse, and S. Das Sarma, Phys. Rev. X 6, 021042 (2016).

[29] J. H. Pixley, D. A. Huse, and S. Das Sarma, Phys. Rev. B 94, 121107 (2016).

[30] J. H. Wilson, J. H. Pixley, P. Goswami, and S. Das Sarma, Phys. Rev. B 95, 155122 (2017).

[31] J. H. Pixley, P. Goswami, and S. Das Sarma, Phys. Rev. Lett. 115, 076601 (2015).

[32] H. Shapourian and T. L. Hughes, Phys. Rev. B 93, 075108 (2016).

[33] S. Bera, J. D. Sau, and B. Roy, Phys. Rev. B 93, 201302 (2016)

[34] T. Holder, C.-W. Huang, and P. M. Ostrovsky, Phys. Rev. B 96, 174205 (2017).

[35] K. Kobayashi, T. Ohtsuki, K.-I. Imura, and I. F. Herbut, Phys. Rev. Lett. 112, 016402 (2014).

[36] S. Liu, T. Ohtsuki, and R. Shindou, Phys. Rev. Lett. 116, 066401 (2016).

[37] W. Pieper and W. Greiner, Z. Phys. Hadrons Nucl. 218, 327 (1969).

[38] W. Greiner, Relativistic Quantum Mechanics. Wave Equations (Springer-Verlag, Berlin, 2000).

[39] D.-H. Lin, Phys. Rev. A 73, 052113 (2006).

[40] M. Buchhold, S. Diehl, and A. Altland, Phys. Rev. B 98, 205134 (2018).

[41] P. Van Mieghem, Rev. Mod. Phys. 64, 755 (1992).

[42] B. Kramer and A. MacKinnon, Rep. Prog. Phys. 56, 1469 (1993).

[43] J. Luttinger and R. Waxler, Ann. Phys. (N.Y.) 175, 319 (1987).

[44] M. C. W. van Rossum, T. M. Nieuwenhuizen, E. Hofstetter, and M. Schreiber, Phys. Rev. B 49, 13377 (1994).

[45] J. Zittartz and J. S. Langer, Phys. Rev. 148, 741 (1966).

[46] S. Yaida, Phys. Rev. B 93, 075120 (2016).

[47] K. Efetov, Supersymmetry in Disorder and Chaos (Cambridge University Press, Cambridge, England, 1997).

[48] E. Brézin, J.-C. L. Guillou, and J. Zinn-Justin, Phys. Rev. D 15, 1558 (1977).

[49] K. Ziegler and A. Sinner, Phys. Rev. Lett. 121, 166401 (2018).

[50] T. Wehling, A. Black-Schaffer, and A. Balatsky, Adv. Phys. 63, 1 (2014). 